\title{
Analysing spatial and temporal dynamics of suicide in South Korea: An application of the dynamic spatial panel data model
}

\author{
Yunho Yeom \\ Department of Public Policy \& Management, Pusan National University, Busan, Republic of Korea
}

\begin{abstract}
This study examines the relationship between the suicide mortality rate and structural covariates in South Korea from 2008 to 2017 under Durkheim's theory of suicide. It applies the dynamic spatial panel data model to explore both spatial and temporal aspects of the suicide phenomena recognising statistical limitations in previous suicide research based on either cross-sectional or longitudinal methodology. The results demonstrate that the suicide mortality rates in South Korea are spatially and temporally dependent on those of neighbouring units or its own time-lagged suicide mortality rates. Moreover, this study estimates that the divorce rate, unemployment rate and land price index, which are assumed to be proxies of social integration and regulation, significantly impact the suicide mortality rates. The results imply that deteriorating socioeconomic conditions are risk factors for the suicide mortality rate and suggests implementing policies to alleviate the high level of social disintegration caused by Korea's deteriorating socioeconomic conditions.
\end{abstract}

\section{Introduction}

Over the past few decades, suicide has been a severe public

Correspondence: Yunho Yeom, Department of Public Policy \& Management, Pusan National University, 2, Busandaehak-ro 63beongil, Geumjeong-gu, Busan, Republic of Korea. Postal code: 46241.

Tel.: +82.51.510.2569 - Fax: +82.51.510.7669

E-mail: veritas4962@pusan.ac.kr

Key words: Suicide; dynamic spatial panel data model; social integration; social regulation; South Korea.

Funding: this work was supported by the Ministry of Education of the Republic of Korea and the National Research Foundation of Korea (NRF-2020S1A5A8045132).

Conflict of interests: the author declares no potential conflict of interests.

Received for publication: 6 December 2020.

Revision received: 11 March 2021.

Accepted for publication: 13 March 2021.

CCopyright: the Author(s), 2021

Licensee PAGEPress, Italy

Geospatial Health 2021; 16:964

doi:10.4081/gh.2021.964 health concern in South Korea since it is the $5^{\text {th }}$ leading cause of death, accounting for approximately 13,000 deaths annually as reported by the Korean Statistical Information Service (KOSIS, 2019). The suicide issue has received substantial academic attention from numerous disciplines, including medical science, psychology and public health. Most suicide research has usually examined individual-level causes of suicide, such as mental disorder or psychological trauma, while few have examined the contextual-level causes of suicide (Cheong et al., 2012; Kang, 2017). A growing number of studies have recently investigated the suicide phenomenon beyond the individual level assuming that the variations of the suicide mortality rate (SMR) reflect the spatial and temporal variations of underlying social conditions across different regions in the country (Yeom, 2019).

Previous studies have consistently demonstrated that SMR is associated with a society's deteriorating socioeconomic conditions under different theoretical backgrounds and methodologies. A volume of contextual-level suicide research has usually operationalised such conditions with measurable indices, such as unemployment rate, divorce rate and demographic structure and found a statistically significant relationship between the SMR and such indices. However, based on either cross-sectional (Baller \& Richardson, 2002; Congdon, 2011) or longitudinal (Phillips, 2013; Nordt et al., 2015) statistical tools, previous suicide research has considered time and space as separate components of the suicide phenomena. Scholars have pointed out that these studies fail to incorporate interaction between space and time into their analyses (Ye \& Wu, 2011; Joo, 2017). They have noted that spatial and temporal analysis becomes essential because it enhances the accuracy of statistical inference and at the same time reduces estimate bias by considering spatial and temporal proximity or dependence (Ye \& Wu, 2011).

Recognising these limitations in traditional statistical models, a growing number of social science studies have used the spatial panel data model, an approach using time series observations of a number of spatial units, to examine the spatial and temporal dynamics of diverse social phenomena, including suicide (Breuer, 2015; Joo, 2017), homicide (Ye \& Wu, 2011) and other social issues (Baltagi et al., 2012; Bussu et al., 2013). These studies not only visualise the spatial and temporal patterns of the investigated phenomena but also extend 'modelling possibilities as compared to the cross-sectional setting for spatial data' as expressed by Ye and $\mathrm{Wu}$ (2011). Despite the utility of the model, few suicide studies in South Korea there have applied the spatial panel data model. This is one of the reasons that this study explores the spatial and temporal dynamics of suicide phenomena in the country using this model. This study first visualises the spatial and temporal variations of suicide phenomena in South Korea by exploratory spatial data analysis (ESDA) using several techniques. Then, it performs spatial panel data regression to examine the impact of deteriorat- 
ing social conditions on the SMR while controlling spatial and temporal dependence. Theoretically, by revisiting Durkheim's seminal work on suicide, this study mainly examines the two types of contextual conditions, such as social integration and regulation, as the factors of protection or risk in relation to suicide.

\section{Socioeconomic covariates of the suicide mortality rate}

As Durkheim (1897) emphasized, the SMR is associated with the underlying social conditions, particularly the level of social integration and regulation. Social integration indicates how actively and constantly individuals are connected, whereas social regulation indicates how strongly society controls individuals by emphasizing norm. When individuals are weakly integrated or social regulation is insufficient, individuals become vulnerable to suicidal ideation or behaviour (Durkheim, 1897; Baller \& Richardson, 2002). Durkheim refers to suicide caused by weak integration as egoistic suicide and suicide caused by insufficient regulation as anomic suicide.

Relying upon Durkheim's notion of egoistic suicide, subsequent research has usually examined the divorce rate as a proxy for low social integration (Stack, 1982; Cheong et al., 2012; Yoon et al., 2015). The SMR and the divorce rate have been positively associated with other contextual predictors, such as economic status indicators (Cheong et al., 2012; Yoon et al., 2015). For instance, Park and Lester (2006), reviewing the divorce rate and the SMR in South Korea between 1983 and 2002, found that the SMR was positively associated with the divorce rate for the elderly populations. However, some research has found negative (Baller \& Richardson, 2002) or non-significant (Bussu et al., 2013) effects of the divorce rate on the SMR. Meanwhile, suicide research has often considered ethnic heterogeneity (Moore et al., 2014) and residential mobility (Baller \& Richardson, 2002) to measure the level of social disintegration. These two indices have also been assumed as indicators of social disorganisation under a criminological theory (Porter \& Purser, 2010). Moreover, Durkheim (1897) pointed out that the lack of social regulation related to dramatic economic and social change is less likely to determine individuals' relative position and the appropriate rewards individuals expect. Whenever radical readjustment necessarily takes place under either faltering or prospering economic conditions, 'individuals feel the torture of unsatisfied passions and are more prone to suicide' (Kang, 2017, p. 5). Predicated on Durkheim's notion of anomic suicide, studies have usually investigated the impact of economic crises on the SMR with different economic indices, including the unemployment rate (Breuer, 2015; Yoon et al., 2015), local tax (Joo, 2017) and the house price index (Eun, 2019). Most studies found a positive impact of deteriorating economic conditions on the SMR, but some found non-significant effects (Bussu et al., 2013).

In summary, previous studies have operationalized Durkheim's two main social forces in explaining suicide (social integration and regulation) with various, available social indices. Mixed effects of such indices on the SMR have been noted depending on the methodology used by each study. However, some indices, including the collective level of employment and marital status, have been found to have a relatively consistent impact on the SMR. Like these previous studies, this study examines the effects of proxies of social integration and regulation on South Korea's SMR by analysing the spatial and temporal dynamics.

\section{Methodology}

\section{Data source and measurement}

Located in East Asia, South Korea has a total area of 100,300 $\mathrm{km}^{2}$ with a population of 51.6 million (The World Bank, 2019). This study's analysis units were 240 municipalities (equivalent to a county in the U.S.) with an average size of $417.9 \mathrm{~km}^{2}$ and an average population of 0.21 million.

From the 1980s, South Korea's economy has been one of the fast-growing countries in the world, invoking a shift from traditional collectivism to western individualism (Kang, 2017). The country has further experienced two major economic crises, in 1998 and 2008, which accompanied drastic economic and social changes. Due to the data availability issue, this study examines suicide phenomena between 2008 and 2017. This study span includes the newer economic crisis in 2008 , which was originally incurred by the U.S. subprime mortgage crisis.

This study used several datasets publicly available at the Korean Statistical Information Service database. First, for the dependent variable, the SMR, this study collected the cause-ofdeath dataset from the annual statistics between 2008 and 2017. The dataset provides the SMR per 100,000 population in each municipality. For the independent variables, (indicators for social integration and socioeconomic conditions) several datasets from the same KOSIS database were collected. The social integration variables included datasets on the divorce rate (measured by the number of divorced populations per 1000 people in each municipality), ethnic heterogeneity (measured by the percentage of the population with non-South Korean nationality) and residential mobility (measured by the percentage of the population who had moved into another municipality). For the socioeconomic variables, this study used datasets on the unemployment rate (measured by the percentage of unemployed population among the population over the age of fifteen) and the land price fluctuation rate (measured by the percentage of the changed price compared to the previous year's price). All these datasets were integrated by municipality in time series covering the period 2008-2017.

\section{Analytical strategy}

In examining the relationship between the SMR and social conditions, this study applied a dynamic spatial panel data model controlling both the spatial dependence between spatial units and the temporal dependence between time series (Belotti et al., 2016; Joo, 2017). The model takes the following form:

$$
Y_{i t}=\tau Y_{i(t-1)}+\psi \sum_{j=1}^{N} w_{i j} Y_{j(t-1)}+\rho \sum_{j=1}^{N} w_{i j} Y_{j t}+X_{i t} \beta+\theta \sum_{j=1}^{N} w_{i j} Z_{i t}+\mu_{i}+\varepsilon_{i t}
$$

where $Y_{i t}$ is the SMR in a spatial unit $(i)$ at a particular time point $(t)$ and the parameter $\tau$ the effect of the lagged SMR at $t-1$. The parameter $\psi$ indicates the spatially weighted average effects of the lagged SMR in adjacent spatial units at $t-1$, whereas the parameter $r$ indicates the spatially weighted average effects of the SMR in adjacent spatial units at $t$. This study created the spatial weight matrix $\left(\mathrm{w}_{\mathrm{ij}}\right)$ based on the contiguity among spatial units. The parameter $\beta$ indicates the effects of the explanatory variables $X_{i t} ; \theta$ the spatially weighted averaged effects of $X_{i t}$ in adjacent spatial units; $\varepsilon_{i t}$ the error; and the parameter $\mu_{i}$ the fixed-effect variant 
under an assumption that $\mu_{i}$ is correlated with the explanatory variables in the model (Belotti et al., 2016).

In addition to the spatial panel data model, ESDA techniques, including Moran's $I$ and the local indicator of spatial association (LISA), were applied to visualize the spatial and temporal variation of the SMR. ESDA techniques are descriptive analysis tools used to depict spatial or temporal patterns of investigated phenomena before examining the dynamic factors to explain their causes (Ye \& Wu, 2011). For instance, ranging from -1 to +1 , Moran's $I$ indicates a global spatial dependence in the SMR, whereas the LISA produces a categorical outcome indicating positive or negative clustering at the local level. This study used the xsmle command in the Stata software package (https:/www.stata.com/) for the dynamic spatial panel data analysis (Belotti et al., 2016) and the QGIS package (https://www.qgis.org/) for data visualization.

\section{Results}

\section{Descriptive statistics}

This study first analysed the characteristics of each variable. The descriptive statistics for variables are presented in Table 1. The first panel presents descriptive statistics by averaging all the observed values between 2008 and 2017, whereas the second panel presents observed values at each time point.

Next, the spatial distribution of the SMR with several ESDA techniques was examined and visualized. First, the spatial distribution of the SMR over time with a series of thematic maps was explored. As seen in Figure 1, the SMR is not evenly distributed but concentrated in South Korea's north-eastern part. Furthermore, these spatial patterns of the SMR have changed during the study span. In particular, the SMR increased sharply right after the U.S subprime mortgage crisis in 2008 plagued the world, including South Korea. Starting from 2008 through 2011, the SMR has steadily increased from 31.57 to 36.41 . Further, after 2012, the SMR decreased consistently to 26.56 in 2017. In 2016 and 2017, no municipality recorded an SMR over 75 . Moreover, other social conditions, such as the unemployment rate or the land price index, have shown a similar pattern of the SMR, which were synchronized with the aftermath of the subprime mortgage crisis (Table 1).

Then, the global spatial dependence among variables with Moran's $I$ statistics was examined. Table 2 presents the spatial dependence at the global level seen over time.

In general, except for residential mobility at particular time points, the spatial dependence for almost all the variables was stable over the study time. In particular, there was a statistically significant spatial autocorrelation with respect to the SMR. The spatial distribution of the SMR in municipalities was far from random, as evidenced by the strong and positive Moran's $I$ statistics. The

Table 1. Descriptive statistics.

\begin{tabular}{|c|c|c|c|c|c|c|c|c|c|}
\hline Variable & & num & Ma: & & Mean & & & Standard d & viation \\
\hline $\begin{array}{l}\text { Outcome variable } \\
\text { Suicide mortality rate (per 100,000 population) }\end{array}$ & & & & & 31.49 & & & 11.6 & \\
\hline $\begin{array}{l}\text { Social integration variables } \\
\text { Divorce rate (per } 1000 \text { population) } \\
\text { Ethnic heterogeneity (\%) } \\
\text { Residential mobility (\%) }\end{array}$ & & & & & $\begin{array}{l}2.15 \\
1.65 \\
1.16\end{array}$ & & & $\begin{array}{l}0.48 \\
1.60 \\
1.71\end{array}$ & \\
\hline $\begin{array}{l}\text { Economic variables } \\
\text { Unemployment rate (\%) } \\
\text { Land price fluctuation rate (\%) }\end{array}$ & & & & & $\begin{array}{l}3.52 \\
1.41\end{array}$ & & & $\begin{array}{l}0.23 \\
1.31\end{array}$ & \\
\hline Variable & 2009 & 2010 & 2011 & 2012 & 2013 & 2014 & 2015 & 2016 & 2017 \\
\hline Suicide mortality rate & 35.99 & 36.01 & 36.41 & 31.97 & 31.65 & 29.73 & 29.32 & 28.37 & 26.56 \\
\hline Divorce rate & 2.38 & 2.32 & 2.19 & 2.19 & 2.20 & 2.21 & 2.09 & 2.06 & 2.05 \\
\hline Ethnic heterogeneity & 1.44 & 1.50 & 1.59 & 1.54 & 1.63 & 1.81 & 1.89 & 1.96 & 1.96 \\
\hline Residential mobility & 1.14 & 1.07 & 0.99 & 0.98 & 0.96 & 1.14 & 1.27 & 1.26 & 1.22 \\
\hline Unemployment rate & 3.60 & 3.70 & 3.40 & 3.20 & 3.10 & 3.50 & 3.60 & 3.70 & 3.70 \\
\hline Land price fluctuation rate & 0.64 & 1.00 & 1.02 & 1.06 & 1.05 & 1.77 & 2.33 & 2.54 & 3.65 \\
\hline
\end{tabular}

Table 2. Moran's I statistics of all variables over study periods.

\begin{tabular}{|c|c|c|c|c|c|c|c|c|c|c|}
\hline Variable & 2008 & 2009 & 2010 & 2011 & 2012 & 2013 & 2014 & 2015 & 2016 & 2017 \\
\hline Suicide mortality rate & $0.39 * * *$ & $0.51^{* * *}$ & $0.43^{* * *}$ & $0.46^{* * *}$ & $0.42 * * *$ & $0.33^{* * *}$ & $0.27 * * *$ & $0.34^{* * *}$ & $0.34^{* * *}$ & $0.27 * * *$ \\
\hline Divorce rate & $0.40 * * *$ & $0.39 * * *$ & $0.17^{* * *}$ & $0.26^{* * *}$ & $0.35^{* * *}$ & $0.32 * * *$ & $0.32 * * *$ & $0.26 * * *$ & $0.32 * * *$ & $0.27 * * *$ \\
\hline Ethnic heterogeneity & $0.27^{* * *}$ & $0.26^{* * *}$ & $0.26 * * *$ & $0.24^{* * *}$ & $0.24^{* * *}$ & $0.25^{* * *}$ & $0.25^{* * *}$ & $0.26^{* * *}$ & $0.28 * * *$ & $0.30 * * *$ \\
\hline Residential mobility & $0.10^{*}$ & $0.10^{*}$ & 0.10 & 0.04 & 0.04 & $0.15^{* *}$ & 0.01 & 0.03 & 0.01 & 0.01 \\
\hline Unemployment rate & $0.11^{*}$ & $0.33^{* * *}$ & 0.05 & $0.34^{* * *}$ & $0.26 * * *$ & $0.44^{* * *}$ & $0.40 * * *$ & $0.32 * * *$ & $0.37 * * *$ & $0.36 * * *$ \\
\hline
\end{tabular}

${ }^{*} \mathrm{P}<0.05 ;{ }^{* *} \mathrm{P}<0.01 ; * * * \mathrm{P}<0.001$ 
positive statistics indicate that when the SMR increased within a municipality, those of neighbouring municipalities also increased. These results support the uneven spatial distribution of the SMR visually observed in Figure 1. In addition to the SMR, Moran's I statistic for other independent variables was statistically significant over time. However, this statistic only presented the spatial dependence at the global level and not where this statistically significant clustering was located. However, this study also examined the potential clustering at the local unit by LISA as seen in Figure 2.

Figure 2 presents the uneven distribution of the SMR at the local level. The High-High relationship (in red) indicated a posi- tive, statistically significant association between the SMR in municipalities and that in neighbouring municipalities, while the high-low and the low-high associations indicated the inverse. Unlike Moran's $I$ statistics in Table 2, the LISA indicators demonstrate that spatial dependence within each variable varies at a more localized level. The existence of spatial dependence among variables, indicated by Moran's I statistics and LISA, is for the descriptive purpose and the subsequent spatial regression analysis (Porter $\&$ Purser, 2010). The existence of spatial dependence indirectly supports applying the spatial panel data model.

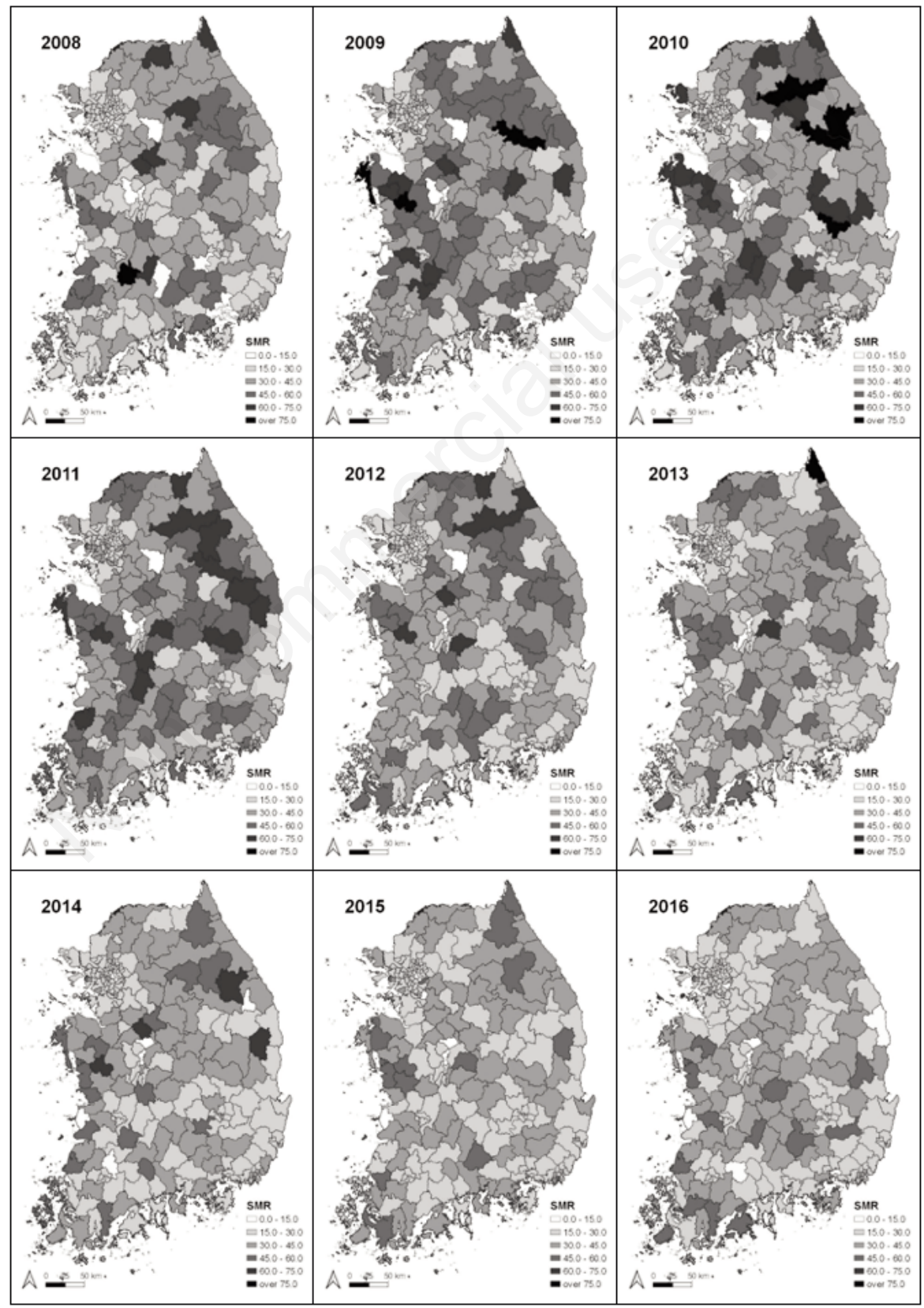

Figure 1. Spatial distribution of the suicide mortality rate (SMR) over time. 


\section{The dynamic spatial panel data model}

This study examined the suicide phenomena between 2008 and 2017 while controlling spatial and temporal dependence by applying the dynamic spatial panel data model. The results are presented in Table 3, which presents the five estimated parameters:

the spatial parameter $(\rho)$; the time-lagged effect without any spatial dependence effect $(\tau)$; the time-lagged effect of the spatially-weighted-average SMR of the neighbouring municipalities $(\psi)$; the relation between structural variables (divorce rate, ethnic heterogeneity, unemployment rate, and land price index) and the SMR $(\beta)$; the spatially weighted averaged effects of structural variables on the $\operatorname{SMR}(\theta)$.
Table 3 shows that there was a significant level of spatial and temporal interaction in the SMR. First, the spatial parameter $(\rho)$, indicating the effect of the spatially weighted dependent variables at time $t$, showed that one unit of SMR increase within a municipality was associated with a 0.09 unit increase of the spatially weighted average SMR of neighbouring municipalities. Also, the two parameters t, and $y$, indicating the time-lagged effect of the SMR at time $t-1$, demonstrated that the SMR at time $t$ was temporally and significantly dependent on the SMR at time $t-1$. The parameter $t$ showed the timelagged effect without any spatial dependence, whereas the parameter $\psi$ indicated the time-lagged effect of spatially-weighted-average SMR of the neighbouring municipalities.

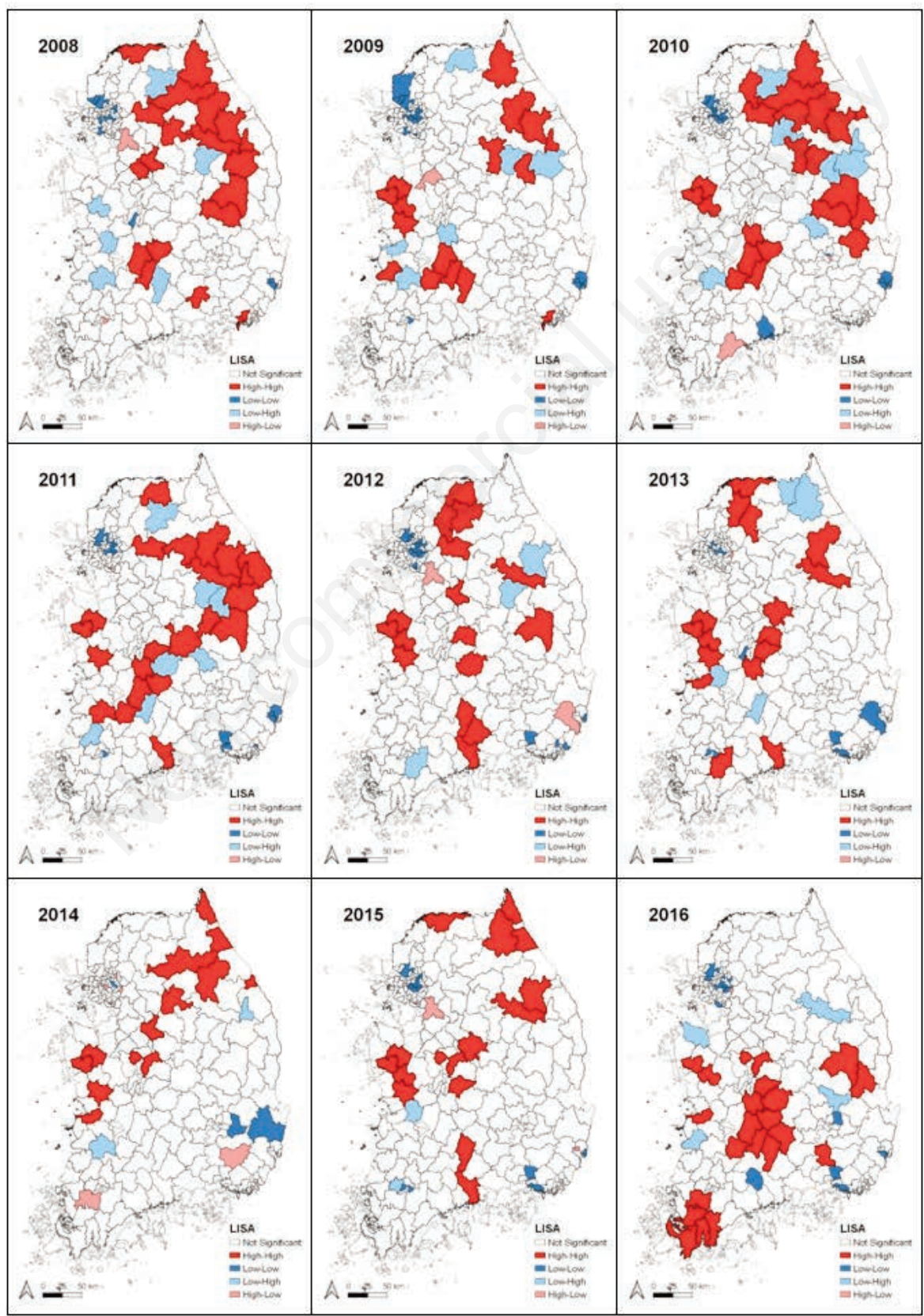

Figure 2. Anselin's local indicator for the spatial association (LISA) for suicide mortality rate over time. 
Furthermore, it was found that the divorce rate and land price fluctuation index significantly impacted the SMR when not controlling the spatial and temporal dependence among several explanatory variables. The parameter $\beta$ demonstrated that one unit increase in the divorce rate was associated with 6.32 units increase of the SMR. In contrast, one unit increase of the land price index was associated with a 1.16 unit decrease of the SMR. On the other hand, when considering the spatial dependence within the set of explanatory variables, the effect of each explanatory variable could change direction and statistical significance. As seen in Table 3, the parameter $\theta$ showed that ethnic heterogeneity, unemployment rate and land price index had significant, but different effects on the SMR.

The dynamic spatial panel data model additionally estimates short- and long-run marginal effects of the explanatory variables on the SMR (Belotti et al., 2016). The marginal effect includes direct, indirect, and total effects. As seen in Table 3, except for two variables, (the ethnic heterogeneity and the residential mobility), the remaining three variables presented statistically significant and fairly consistent marginal effects on the SMR. In particular, the negative impact of the land price index on the SMR was found to be consistent across all short- and long-run direct and indirect marginal effects. Lastly, this study performs a post-estimation test to examine the difference between observed and predicted values. As seen in Figure 3, this study found that the dynamic spatial panel data model's estimation is fairly consistent with the actual suicide phenomena in South Korea.

\section{Discussion}

Recognising the lack of suicide studies conducted at the contextual level in South Korea, this study examined the effects of socioeconomic conditions on the SMR. In particular, based on Durkheim's theory of suicide, the effect of social integration and regulation on SMR was investigated. Methodologically, this study applied the dynamic spatial panel data model to control both spatial and temporal autocorrelation inherent in social phenomena. As a result, it was found that the distribution of SMR in South Korea is both spatially and temporally dependent. It was estimated that the spatially weighted SMR of neighbouring municipalities impacted the SMR in a municipality at a time point (t), time point $(t)$ as well as a previous time point $(t-1)$. These results are consistent with previous studies that also found such autocorrelation within suicide phenomena (Bussu et al., 2013; Joo, 2017). These results indicate that suicide in Korea is a phenomenon that diffuses over neighbouring municipalities and consistently persist, coupled with the underlying social structure and conditions.

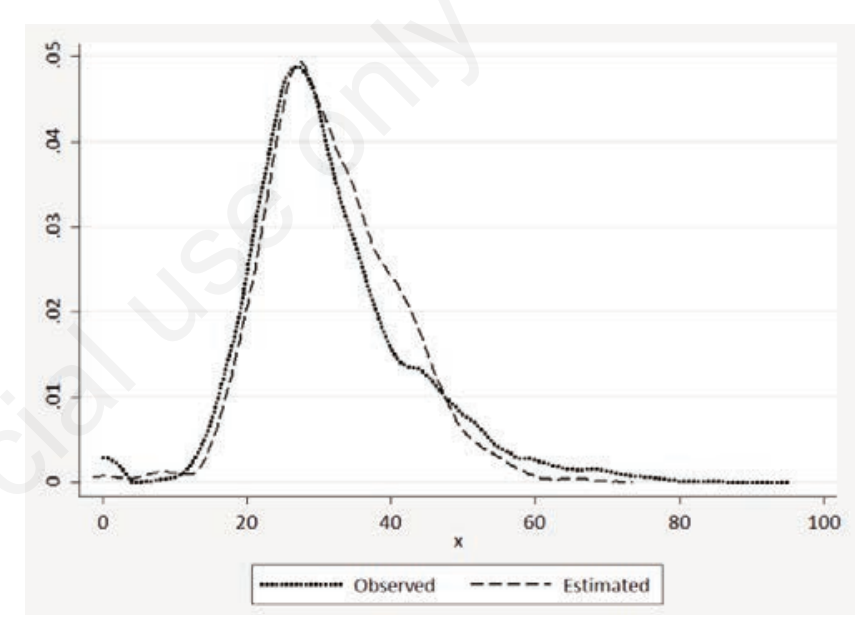

Figure 3. xsmle post-estimation testing of the observed and estimated values.

Table 3. Results of the dynamic spatial panel data model.

\begin{tabular}{|c|c|c|c|c|c|c|c|c|c|}
\hline Structural covariate & $\begin{array}{l}\text { Lagged } \\
\text { effect }\end{array}$ & $\begin{array}{c}X \\
(\beta)\end{array}$ & $\begin{array}{l}\text { WX } \\
(\theta)\end{array}$ & $\begin{array}{c}\text { SR } \\
\text { direct }\end{array}$ & $\begin{array}{c}\text { SR } \\
\text { indirect }\end{array}$ & $\begin{array}{c}\text { SR } \\
\text { total }\end{array}$ & $\begin{array}{l}\text { LR } \\
\text { direct }\end{array}$ & $\begin{array}{c}\mathrm{LR} \\
\text { indirect }\end{array}$ & $\begin{array}{l}\mathrm{LR} \\
\text { total }\end{array}$ \\
\hline $\begin{array}{l}\operatorname{SMR}(t-l)(\tau) \\
\text { Weighted }\end{array}$ & $0.18^{* * *}$ & - & - & - & - & - & - & - & - \\
\hline $\operatorname{SMR}(t-1)(\Psi)$ & $0.16^{* * *}$ & - & - & - & - & - & - & - & - \\
\hline $\begin{array}{l}\text { Divorce rate } \\
\text { Ethnic }\end{array}$ & - & $6.32^{* * *}$ & -0.59 & $6.34^{* * *}$ & -0.01 & $6.33^{* * *}$ & $7.87^{* * *}$ & 1.63 & $9.50^{* * *}$ \\
\hline $\begin{array}{l}\text { Heterogeneity } \\
\text { Residential }\end{array}$ & - & 0.52 & $-1.48^{*}$ & 0.50 & $-1.21^{*}$ & -0.71 & 0.49 & $-1.66^{*}$ & -1.15 \\
\hline Mobility & - & 0.04 & 0.37 & 0.05 & 0.31 & 0.36 & 0.10 & 0.47 & 0.57 \\
\hline $\begin{array}{l}\text { Unemployment } \\
\text { Rate } \\
\text { Land price }\end{array}$ & - & 0.13 & $1.14^{* * *}$ & 0.17 & $0.96^{* * *}$ & $1.13^{* * *}$ & 0.32 & $1.45^{* * *}$ & $1.77^{* * *}$ \\
\hline Fluctuation rate & - & $-1.16^{* * *}$ & $-0.67^{*}$ & $-1.19 * * *$ & $-0.64^{* *}$ & $-1.83^{* * *}$ & $-1.55^{* * *}$ & $-1.26 * * *$ & $-2.81^{* * *}$ \\
\hline $\begin{array}{l}\text { Spatial parameter ( } \rho) \\
\text { Variance } \\
\text { Fixed-effect mean } \\
\text { R-quare (within) } \\
\text { R-Square (between) } \\
\text { R-Square (overall) }\end{array}$ & & & & & $\begin{array}{c}0.09 * * * \\
54.49 * * * \\
9.18 \\
0.19 \\
0.52 \\
0.36\end{array}$ & & & & \\
\hline
\end{tabular}

$\mathrm{X}(\beta)$, effect of independent variable; $\mathrm{WX}(\theta)$, spatially weighted effect of independent variable; $\mathrm{SR}$, sort-run; $\mathrm{LR}$, long-run; $\mathrm{SMR}$, suicide mortality rate. ${ }^{*} \mathrm{P}<0.05 ;{ }^{* *} \mathrm{P}<0.01 ;{ }^{* * *} \mathrm{P}<0.001$. 
Specifically, this study found that among several indicators of social integration and regulation, divorce rate, unemployment rate and land price fluctuation had a relatively persistent impact on the SMR. As previous researchers noted, the divorce rate is one of 'the strongest predictors of the suicide rate' in South Korea (Park \& Lester, 2006). Divorce itself is an individual-level life event accompanying a high level of psychological distress, and on the aggregate level, it leads to the family dissolution and social isolation, which eventually weakens the level of social integration under Durkheim's theory (Bussu et al., 2013). Also, even though this study did not examine a cultural covariate of SMR, several scholars have pointed out that conflicts between traditional Confucian collectivism and western individualism in South Korea have blurred the social norm and values that individuals collectively pursue. As noted by Kang (2017), these underlying cultural conflicts 'eroded the strength of individual bonds and ties to social groups'. Moreover, along with the impact of the cultural conflicts on the SMR, these conflicts can alter the direction and strength of the impact on the SMR of other social indices, including divorce and unemployment. Some scholars have pointed out that social perspectives on divorce and unemployment vary from culture to culture (Adamczyk, 2013; Eichhorn, 2014), and this suggests that in societies with different cultural perspectives, the impact of these factors on SMR can differ. Future studies need to incorporate possible cultural covariates of SMR into their analyses.

Moreover, this study found that the unemployment rate have a positive impact on the SMR, which is congruent with previous findings (Breuer, 2015). Durkheim (1897) pointed out that drastic economic and social changes that lead to insufficient social regulation make individuals prone to suicide. In particular, the economic downturn incurred by the U.S. subprime mortgage crisis in 2008 resulted in a sudden surge in the unemployment rate in South Korea that, in turn, made populations vulnerable to suicide. High unemployment rate levels disturb the social regulation's equilibrium by disrupting the relative position of individuals and the rewards that they expect. After the latest economic crisis that started in 2008, the SMR declined and returned to being as it was due to regained equilibrium of social regulation. On the other hand, the changing land price index impacted the SMR in South Korea negatively and consistently. A rise in land prices is usually associated with economic prosperity, which Durkheim assumes to be another type of economic change that possibly has a positive impact on the SMR. However, this study indicates that the land price rise during the study span could not be considered the sudden growth that requires a serious readjustment in the social order. Rather, the rise in land price directed the relative position of individuals and determined the appropriate rewards that individuals expect in society as long as the rise is within the boundary of society's economic and moral ideals (Kang, 2017). In other words, the level of rising inland prices observed in this country during the study span seems to be moderate, not enough to collectively put the populations into an anomic state.

As scholars have consistently pointed out, some parts of South Korea showed a discriminatorily sharp increase in land price during the past few decades, resulting in residential or economic polarization (Kim et al., 2020). In these localized areas, the high level of inequality and other social indices indicating social deprivations significantly impact public health, including the SMR, in the long run (Moon \& Chung, 2019). Thus, future studies need to use methodologies to explore the long-term association between the inequality level and these phenomena at the more localized lev- els. Despite the findings and policy implications, this study has some limitations. This study analysed the SMR of the entire Korean population. However, as previous research have pointed out, the SMR of different demographic subgroups, such as gender groups and elderly populations, have shown different trends (Kang, 2017; Joo, 2017). Future studies need to investigate genderand/or age-specific SMR to understand suicide phenomena in the more stratified spectrum. Such studies can propose more narrowly customized suicide intervention programmes to meet the specific population's specific needs. Furthermore, even though this study examined the effects of two social forces suggested by Durkheim with available social indices, it might have omitted other possible SMR covariates, e.g., population-level alcohol consumption (Yeom, 2019). Thus, future studies need to include more appropriate variables, such as the Gini coefficient (Hussain, 2018; Tran \& Morrison, 2020), percentage of population below poverty level and church attendance rate, which can impact the SMR (Baller \& Richardson, 2002).

\section{Conclusions}

The results of this study provide support for policymakers to develop diverse suicide prevention programmes at the municipality level. In particular, given that the suicides spatially cluster and temporally persist at the local level, national policymakers need to disproportionately allocate available resources depending on the level of SMR in vulnerable municipalities. Additionally, considering that the SMR is associated with the level of social integration, municipalities with a high level of SMR need to establish a local community centre providing community members the opportunity to mingle with each other and giving healthcare workers appropriate training. This would develop social workers' ability to assist and communicate with vulnerable populations.

\section{References}

Adamczyk A, 2013. The effect of personal religiosity on attitudes toward abortion, divorce, and gender equality: Does cultural context make a difference? EurAmerica 43:213-53.

Baller RD, Richardson KK, 2002. Social integration, imitation, and the geographic patterning of suicide. Am Sociol Rev 67:873-88.

Baltagi BH, Blien U, Wolf K, 2012. A dynamic spatial panel data approach to the German wage curve. Econ Model 29:12-21.

Belotti F, Hughes G, Mortari AP, 2016. Spatial panel data models using Stata. Stata J 17:139-80.

Breuer C, 2015. Unemployment and suicide mortality: Evidence from regional panel data in Europe. Health Econ 24:936-50.

Bussu A, Detotto C, Sterzi V, 2013. Social conformity and suicide. J Socio Econ 42:67-78.

Cheong KS, Choi MH, Cho BM, Yoon TH, Kim CH, Kim YM, Hwang IK, 2012. Suicide rate differences by sex, age, urbanicity, and related regional factors in Korea. J Prev Med Public Health 45:70-7.

Congdon P, 2011. The spatial pattern of suicide in the U.S. in relation to deprivation, fragmentation and rurality. Urban Stud 48:2101-22.

Durkheim E, 1897. Suicide. Free Press, Glencoe, IL, USA. 
Eichhorn J, 2014. The (non-) effect of unemployment benefits: Variations in the effect of unemployment on life-satisfaction between EU countries. Soc Indic Res 119:389-404.

Eun S, 2019. Contextual association between political regime and adolescent suicide risk in Korea: A 12-year repeated cross-sectional study from Korea. Int J Environ Res Public Health 16:874-887.

Hussain M, 2018. Do suicide rates correlate with the Gini coefficient \& GDP? Aus Aca Acc \& Fin Rev 4:11-4.

Joo Y, 2017. Spatiotemporal study of elderly suicide in Korea by age cohort. Public Health 142:144-51.

Kang T, 2017. Suicide in South Korea: revisiting Durkheim's suicide. J Soc Thought 2:3-14.

Kim JN, Han GH, Ro SH, 2020. A study on the residential environment polarization and characteristics in Seoul. Resident Environ 18:43-59 [Article in Korean].

Korean Statistical Information Service (KOSIS). 2019. Suicide statistics. Available from: http://kosis.kr/

Moon DS, Chung HJ, 2019. Two economic crises, unemployment, working poor, and gender: Explaining the dynamics of the risk patterns of suicide in South Korea. J Korean Welfare State Soc Policy 3:28-53.

Moore MD, Recker NL, Heirigs M, 2014. Suicide and the creative class. Soc Indic Res 119:1613-26.

Nordt C, Warnke I, Seifritz E, Kawohl W, 2015. Modelling suicide and unemployment: A longitudinal analysis covering 63 coun- tries, 2000-11. Lancet Psychiat 2:239-45.

Park BCB, Lester D, 2006. Social integration and suicide in South Korea. Crisis 27:48-50.

Phillips JA, 2013. Factors associated with temporal and spatial patterns in suicide rates across U.S. states, 1976-2000. Demography 50:591-614.

Porter JR, Purser CW, 2010. Social disorganization, marriage, and reported crime: a spatial econometrics examination of family formation and criminal offending. J Crim Justice 38:942-50.

The World Bank, 2019. The Republic of Korea data. Available from: https://data.worldbank.org/country/korea-rep Accessed: 30 May 2020.

Tran F, Morrison C, 2020. Income inequality and suicide in the United States: a spatial analysis of 1684 U.S. counties using geographically weighted regression. Spat Spatio-Temporal Epidemiol 34:1-9.

Ye X, Wu L, 2011. Analyzing the dynamics of homicide patterns in Chicago: ESDA and spatial panel approaches. Appl Geogr 31:800-7.

Yeom Y, 2019. Population-level alcohol consumption and suicide mortality rate in South Korea: An application of multivariable spatial regression model. Geospat Health 14:163-70.

Yoon T, Noh M, Han J, Jung-Choi K, Khang Y, 2015. Deprivation and suicide mortality across 424 neighborhoods in Seoul, South Korea: A Bayesian spatial analysis. Int J Public Health 60:969-76. 